\title{
Concordancia en el diagnóstico entre pediatría y salud mental
}

\section{Diagnostic concordance between paediatric and mental health}

\author{
N. Landa ${ }^{1}$, A. Goñi ${ }^{2}$, E. García de Jalón ${ }^{3}$, J.J. López-Goñi ${ }^{4}$
}

\section{RESUMEN}

Objetivo. Analizar el grado de concordancia en el diagnóstico entre los profesionales de los servicios de pediatría y de salud mental.

Pacientes y métodos. El trabajo se ha realizado con 207 pacientes de 0 a 16 años, derivados desde pediatría al centro de Salud Mental (CSM) de Estella durante los años 2006 y 2007. Se calcula el índice de concordancia Kappa global y específico para los diferentes diagnósticos mediante Epidat 3.1.

Resultados. El índice Kappa global de concordancia en el diagnóstico entre ambos niveles asistenciales es de 0,58 . Existe una importante variabilidad en la concordancia obtenida para los diferentes diagnósticos. La concordancia es débil $(0,2-0,4)$ para el trastorno específico del desarrollo, los trastornos depresivos y los adaptativos. Se obtiene una concordancia moderada $(0,41-0,6)$ para el retraso mental, el trastorno generalizado del desarrollo, los códigos $\mathrm{Z}$ y el trastorno de rivalidad entre hermanos (celos). La concordancia es buena $(0,61-0,8)$ para el trastorno por déficit de atención e hiperactividad, el trastorno de ansiedad y el trastorno disocial. Por último, la concordancia diagnóstica es muy buena $(>0,8)$ para el trastorno del control de esfínteres y para el trastorno de la conducta alimentaria.

Conclusiones. La concordancia en el diagnóstico alcanzado entre los servicios de pediatría y el centro de salud mental es moderada. Existe una importante variabilidad en la concordancia obtenida para los diferentes diagnósticos.

Palabras clave. Atención primaria pediátrica. Salud mental. Concordancia diagnóstica. Derivaciones.

\begin{abstract}
Background. To analyse the diagnostic concordance between the paediatric and mental health (MH) services.

Materials and methods. Two hundred and seven patients from 0 to 16 years referred from paediatrics to the Estella Mental Health Centre during 2006 and 2007. Concordance between global Kappa Index and specific diagnosis was calculated with Epidat 3.1. An analysis was made for each diagnostic category of the percentage of cases where the diagnosis made in paediatrics was confirmed in Mental Health.
\end{abstract}

Results. The global diagnostic concordance between both medical care levels has a Kappa Index of 0.58 . There is a wide variability in the concordance between the different diagnoses. The concordance is weak (0.2-0.4) for specific developmental disorder, affective disorders and adaptative disorders. A moderate concordance (0.41-0.6) is obtained for mental retardation, pervasive developmental disorder, z diagnostics, and sibling rivalry disorder. Concordance is good for attention deficit disorder with hyperactivity, anxiety disorder and conduct disorder. Finally, the diagnostic concordance is very good for enuresis and encopresis and for eating disorders.

Conclusions. The diagnostic concordance obtained between paediatric services and the mental health centre is moderate. A wide variability is obtained in the concordance between different diagnoses.

Key words. Paediatric primary care. Mental health. Diagnostic concordance. Referrals.

\section{Correspondencia}

Natalia Landa González

Centro de Salud Mental de San Juan

Avda. Barañáin, 14

31008 Pamplona

Tfno. 948190040-616737911

Fax 948190043

E-mail: Natalia.landa.gonzalez@cfnavarra.es 


\section{INTRODUCCIÓN}

La enfermedad mental es un problema de salud pública de primera magnitud. Supone un elevado porcentaje de discapacidad, por lo que su detección, diagnóstico y tratamiento precoz resulta fundamental en su pronóstico ${ }^{1}$.

Los estudios epidemiológicos avalan la existencia de una elevada prevalencia de trastornos mentales en población infantojuvenil. En una revisión ${ }^{2}$ de los estudios epidemiológicos sobre psicopatología en niños y adolescentes se obtiene una prevalencia media en población general de $15,8 \%$. Destaca la amplia variabilidad entre los diferentes estudios, con un rango entre el 1 y el $51 \%$.

Asimismo, se estima que la presencia de trastornos mentales entre los pacientes atendidos en una consulta de pediatría resulta muy elevada ${ }^{3-6}$. Por este motivo, los pediatras tienen un papel clave tanto en la detección de los trastornos emocionales y conductuales en la infancia, como en la derivación a servicios especializados de salud mental ${ }^{4,7,8}$.

En los últimos años se ha incrementado el interés y la atención de los pediatras por los problemas de salud mental ${ }^{5}$. Sin embargo, la atención integral a este tipo de trastornos supera las posibilidades y objetivos de la intervención en la consulta ambulatoria de pediatría ${ }^{4,5}$. Por ello, la adecuada coordinación con los centros de salud mental que asumen la demanda es un factor clave para favorecer el tratamiento de estos trastornos.

La utilización de un lenguaje común basado en los manuales de clasificación aceptados por consenso como la Clasificación de los trastornos mentales y del comportamiento (CIE-10) de la Organización Mundial de la Salud ${ }^{9}$ o el DSM-IV-TR de la APA ${ }^{10}$ resulta, a pesar de sus múltiples limitaciones, el punto de partida que posibilita una adecuada comunicación y favorece la coordinación entre ambos niveles asistenciales.

No obstante, destaca la ausencia de investigaciones que estudien la concordancia en el diagnóstico entre los servicios de pediatría y los centros de salud mental. De hecho, no ha sido posible localizar, utilizando las bases de datos habituales, un estudio de las mismas características. La inexistencia en la mayor parte de Europa, y especialmente en el entorno anglosajón, de la atención pediátrica específica desde los servicios de atención primaria, puede explicar esta carencia de trabajos específicos sobre el tema.

Como excepción, existe un estudio realizado en España ${ }^{3}$, que analiza la validez del diagnóstico clínico de trastorno por déficit de atención con hiperactividad (TDAH) en las derivaciones realizadas desde pediatría a psiquiatría. En esta investigación, se obtiene una validez diagnóstica moderada, con una especificidad baja, ya que tan sólo en el 36\% de los casos derivados como sospecha de TDAH se confirmó el diagnóstico en psiquiatría.

Por otra parte, en un estudio sobre concordancia diagnóstica realizado con población adulta en la misma zona de salud y el mismo $\mathrm{CSM}^{11}$ que la actual investigación, se obtuvo una concordancia global débil (Kappa: 0,38) con marcadas diferencias entre los distintos diagnósticos. Destacaba la escasa concordancia obtenida para los trastornos adaptativos (Kappa: 0,19). Este dato venía a confirmar hallazgos previos en población adulta, que encontraban una escasa identificación de los trastornos adaptativos en atención primaria ${ }^{12-14}$.

Así, el objetivo de este trabajo es valorar el grado de acuerdo en el diagnóstico de los casos derivados, entre los servicios de pediatría que realizan la detección y la valoración inicial y el centro de Salud Mental que posteriormente evalúa, diagnostica $\mathrm{y}$ trata los trastornos mentales.

\section{MATERIAL Y MÉTODOS}

\section{Pacientes}

\section{Criterios de inclusión}

- Pacientes infanto-juveniles (IJ), de 0 a 16 años, derivados desde pediatría al centro de Salud Mental (CSM) de 
Estella por primera vez durante los años 2006 y 2007.

- Pacientes, atendidos en el CSM con anterioridad, que son derivados de nuevo por el pediatra tras un período de al menos 6 meses desde el alta.

\section{Criterios de exclusión}

- Casos en los que se determinó la ausencia de trastorno psiquiátrico. Fueron 7 pacientes en los que en el volante de derivación no constaba descripción de la sintomatología ni diagnóstico, sino que eran derivados por petición de la familia. En todos los casos tras realizar la primera consulta se descartó la presencia de trastorno psiquiátrico y se les dio el alta.

- Casos derivados desde otros servicios especializados. En concreto, se trató de 8 sujetos derivados desde la Unidad de Hospitalización Psiquiátrica y desde el Hospital de Día Infanto-Juvenil.

Finalmente, la muestra objeto de estudio quedó constituida por 207 sujetos.

\section{Contexto}

Este CSM, uno de los 9 que componen la red de Salud Mental de Navarra, se encuentra en una zona rural y atiende a una población de referencia de 8.976 menores de 16 años. En la actualidad, la salud mental se sitúa dentro de la atención sanitaria especializada por lo que el acceso no es directo, sino que se realiza a través de atención primaria o de otros servicios especializados. Las derivaciones fueron realizadas por 8 pediatras, distribuidos en 8 áreas de salud. La atención en el CSM la realizan dos psicólogas clínicas y dos médicas psiquiatras, si bien el diagnóstico inicial es llevado a cabo en todos los casos infanto-juveniles por las psicólogas.

\section{Procedimiento}

Inicialmente, se recogió la aproximación diagnóstica formulada por el pedia- tra en el volante de derivación. Posteriormente, se registró el diagnóstico realizado en salud mental mediante una entrevista clínica y utilizando los criterios diagnósticos de la clasificación CIE-10. Como se ha señalado, la evaluación y el diagnóstico de los casos derivados los realizaban las dos psicólogas clínicas del CSM, responsables del programa infanto-juvenil.

Para simplificar el análisis de los resultados, se redujeron las categorías diagnósticas a las siguientes: retraso mental, trastorno específico del desarrollo, Trastorno Generalizado del Desarrollo (TGD), Trastorno por Déficit de Atención e Hiperactividad (TDAH), Trastorno de Ansiedad, Trastorno de la Eliminación (encopresis y enuresis), Trastorno de la Conducta Alimentaria (TCA), Trastorno adaptativo, Trastorno depresivo, Trastorno psicótico, Trastorno disocial, Código Z, Celos y Otros. Esta última categoría incluye los diagnósticos: mutismo, trastornos por uso de sustancias, trastornos del sueño y tricotilomanía, que se agruparon debido a su escasa prevalencia en la muestra.

El análisis de los datos se realizó en dos fases. En primer lugar, se calculó el índice de concordancia Kappa global y para los diferentes trastornos, entre los diagnósticos realizados en los servicios de pediatría y los llevados a cabo por profesionales de salud mental, mediante el programa estadístico Epidat 3.1. Para la interpretación de este índice, se consideró el modelo de referencia de Altman ${ }^{15}$.

En segundo lugar, se analizó el número de casos en los que coincidía el diagnóstico de pediatría y de salud mental, la proporción de diagnósticos realizados desde pediatría que se confirman en salud mental, junto con el porcentaje de cada trastorno que fue identificado adecuadamente desde pediatría.

\section{RESULTADOS}

El Índice Kappa global de concordancia en el diagnóstico entre ambos niveles asistenciales es 0,58 , lo que corresponde a una concordancia moderada. 
Tabla 1. Concordancia en el diagnóstico para los diferentes diagnósticos.

\begin{tabular}{|l|c|c|c|c|c|}
\hline \multicolumn{1}{|c|}{ Trastornos } & Kappa & EE & IC (95\%) & Z & p \\
\hline T. Eliminación & 0,93 & 0,07 & $0,8-1$ & 13,46 & 0,000 \\
\hline TCA & 0,92 & 0,08 & $0,77-1$ & 13,32 & 0,000 \\
\hline T. Ansiedad & 0,72 & 0,06 & $0,6-0,83$ & 11,05 & 0,000 \\
\hline TDAH & 0,68 & 0,06 & $0,57-0,80$ & 10,49 & 0,000 \\
\hline T. Disocial & 0,68 & 0,11 & $0,45-0,90$ & 9,93 & 0,000 \\
\hline TGD & 0,57 & 0,22 & $0,13-1$ & 9,12 & 0,000 \\
\hline Retraso mental & 0,56 & 0,22 & $0,12-1$ & 8,26 & 0,000 \\
\hline Celos & 0,49 & 0,22 & $0,06-0,91$ & 7,37 & 0,000 \\
\hline T. Específico desarrollo & 0,40 & 0,12 & $0,17-0,65$ & 6,22 & 0,000 \\
\hline T. Depresivos & 0,40 & 0,09 & $0,21-0,58$ & 6,16 & 0,000 \\
\hline Códigos Z & 0,35 & 0,15 & $0,05-0,66$ & 5,66 & 0,000 \\
\hline T. Adaptativos & 0,34 & 0,10 & $0,14-0,55$ & 5,26 & 0,000 \\
\hline Psicosis & 0,00 & 0,00 & $0-0$ & 0,00 & 1,000 \\
\hline Total & $\mathbf{0 , 5 8}$ & $\mathbf{0 , 0 4}$ & $\mathbf{0 , 5 1 - 0 , 6 6}$ & $\mathbf{2 2 , 1 8}$ & $\mathbf{0 , 0 0 0}$ \\
\hline
\end{tabular}

T. = Trastorno; TCA = Trastorno de la conducta alimentaria; TDAH = Trastorno por déficit de atención con hiperactividad; TGD = Trastorno generalizado del desarrollo.

En la tabla 1 se presenta la concordancia para los diferentes diagnósticos. La concordancia diagnóstica es muy buena $(>0,8)$ para los trastornos de la eliminación y para el trastorno de la conducta alimentaria. La concordancia es buena $(0,61-0,8)$ para los trastornos de ansiedad, el trastorno por déficit de atención con hiperactividad, y el trastorno disocial. Se obtiene una concordancia moderada $(0,41-0,6)$ para el trastorno generalizado del desarrollo, el retraso mental y el trastorno de rivalidad entre hermanos (celos). En cambio, para el trastorno específico del desarrollo, los trastornos depresivos, los códigos $\mathrm{Z}$ y los trastornos adaptativos la concordancia es débil $(0,2-0,4)$. Por último, la concordancia es muy pobre para los trastornos psicóticos, aunque el escaso número de casos detectados (2) obliga a considerar este dato con cautela.

En la tabla 2 se presenta la comparación cualitativa entre los diagnósticos realizados por pediatras y los que finalmente se llevan a cabo en salud mental. En el TGD y los trastornos de la eliminación se con- firman la totalidad de los diagnósticos. En cuanto al TCA, el TDAH, el retraso mental, los trastornos de ansiedad, el trastorno disocial y el trastorno por celos el diagnóstico se confirma en al menos dos tercios de los casos. En los códigos Z, los trastornos específicos del desarrollo y los trastornos depresivos, el porcentaje de coincidencia disminuye, aunque sigue situándose por encima del $50 \%$. La excepción se sitúa en el trastorno adaptativo, ya que tan sólo en el $36 \%$ de los casos se confirma el diagnóstico de pediatría. Al no existir ningún diagnóstico de trastorno psicótico en pediatría, no ha podido ser confirmado ningún caso.

Desde otra perspectiva, se analiza el porcentaje de cada diagnóstico que ha sido identificado adecuadamente en atención primaria. En este caso, destacan el TCA como el trastorno mejor identificado con la totalidad de los casos filiados correctamente. A continuación, se sitúan los trastornos de ansiedad, los trastorno de la eliminación, el TDAH y el trastorno disocial, con un alto porcentaje de identificación 
(superior al 70\%). En cambio, en el retraso mental, el trastorno adaptativo, el TGD, el trastorno por celos y el trastorno depresivo el porcentaje de detección disminuye situándose entre el 50 y el $40 \%$. Por otra parte, los trastornos específicos del desarrollo y los códigos $\mathrm{Z}$, son los peor identificados, con un 37 y un $27 \%$, respectivamente. Por último, en los trastornos psicóticos, no se detecta el trastorno en ninguno de los dos casos diagnosticados en Salud Mental (Tabla 2).

Tabla 2. Distribución de los diagnósticos y porcentajes de coincidencia.

\begin{tabular}{|c|c|c|c|c|c|}
\hline & \multicolumn{2}{|c|}{ Diagnósticos } & \multicolumn{2}{|c|}{$\begin{array}{c}\text { Confirmación } \\
\text { diagnósticos }\end{array}$} & \multirow{2}{*}{$\begin{array}{c}\begin{array}{c}\text { Detección } \\
\text { correcta en } \\
\text { AP }\end{array} \\
\%\end{array}$} \\
\hline & (N) & (N) & (N) & $\%$ & \\
\hline TGD & 2 & 5 & 2 & $100 \%$ & $40 \%$ \\
\hline T. Eliminación & 7 & 8 & 7 & $100 \%$ & $87 \%$ \\
\hline TCA & 7 & 6 & 6 & $86 \%$ & $100 \%$ \\
\hline TDAH & 55 & 46 & 38 & $69 \%$ & $83 \%$ \\
\hline Retraso Mental & 3 & 4 & 2 & $67 \%$ & $50 \%$ \\
\hline T. Ansiedad & 51 & 37 & 34 & $67 \%$ & $92 \%$ \\
\hline T. Disocial & 12 & 11 & 8 & $67 \%$ & $72 \%$ \\
\hline Celos & 3 & 5 & 2 & $67 \%$ & $40 \%$ \\
\hline Otros* & 7 & 8 & 5 & $67 \%$ & $62 \%$ \\
\hline Códigos Z & 5 & 11 & 3 & $60 \%$ & $27 \%$ \\
\hline T. Específico desarrollo & 11 & 16 & 6 & $54 \%$ & $37 \%$ \\
\hline T. Depresivo & 22 & 30 & 12 & $54 \%$ & $40 \%$ \\
\hline T. Adaptativo & 22 & 18 & 8 & $36 \%$ & $44 \%$ \\
\hline Psicosis & 0 & 2 & 0 & $0 \%$ & $0 \%$ \\
\hline Total & 207 & 207 & 133 & & \\
\hline
\end{tabular}

*Incluye: mutismo n: 1, consumo tóxicos n: 2, trastornos del sueño n: 3 y tricotilomanía n: 2.

$\mathrm{TGD}=$ Trastorno generalizado del desarrollo; $\mathrm{T}$. = Trastorno; TCA = Trastorno de la conducta alimentaria;

TDAH = Trastorno por déficit de atención con hiperactividad.

\section{DISCUSIÓN}

Los datos obtenidos permiten afirmar que la coincidencia en el diagnóstico entre ambos niveles asistenciales es elevada. Aunque la concordancia global es tan sólo moderada, cuando se considera la concordancia para cada diagnóstico encontramos que en 5 de las 13 categorías diagnósticas la concordancia es buena o muy buena, con tan sólo 3 trastornos con una concordancia débil (aunque alta dentro de este rango).

Como ya se ha señalado, dado lo novedoso del tema estudiado, carecemos de datos para la comparación de los actuales hallazgos con otros previos en una población equiparable.

En un estudio similar realizado por el mismo equipo investigador con población adulta $^{11}$, se obtuvo un nivel considerablemente inferior de acuerdo diagnóstico, ya que el índice Kappa global era de 0,385. Asimismo, existía una mayor heterogeneidad en la concordancia obtenida para los diferentes diagnósticos, por lo que los índices Kappa se distribuían en un rango más amplio $(0,18-0,91)$. Sin embargo, sí se repite en ambos estudios la escasa concordancia 
en el diagnóstico de los trastornos adaptativos, que se situaba en un índice Kappa de 0,19 en el estudio de adultos, y es la más baja de todos los trastornos en el actual estudio $(0,34)$. Tan sólo se identifican adecuadamente el $44 \%$ (en pediatría) y el $26 \%$ (en medicina de familia) de los casos que finalmente se diagnostican como trastornos adaptativos en salud mental. Así, parece que en general, los profesionales de atención primaria presentan dificultades para identificar adecuadamente los trastornos adaptativos. Estos resultados son avalados por los obtenidos por otros autores en población adulta ${ }^{12-14}$.

Por otra parte, resulta sorprendente la elevada concordancia obtenida en el diagnóstico del TDAH (Kappa=0,68), ya que el diagnóstico se confirma en un $69 \%$ de los casos. Este dato es especialmente llamativo dadas las importantes dificultades diagnósticas de este trastorno ${ }^{16,17}$. De hecho, un estudio sobre la calidad del diagnóstico de TDAH en pediatría desarrollado en España, obtiene porcentajes de coincidencia muy inferiores, de forma que tan sólo se confirmaba el diagnóstico en un 36\% de los casos derivados como $\mathrm{TDAH}^{3}$. Una posible explicación de este fenómeno, además del creciente interés existente en pediatría por este trastorno ${ }^{5}$, es la existencia en Navarra desde 2002 de un protocolo de coordinación entre el centro escolar y pediatría para la derivación de los posibles casos de TDAH. Según este protocolo, el orientador escolar debe evaluar inicialmente al niño, lo que incluye una entrevista con los profesores y con los padres, con aplicación de cuestionarios. A partir de esta valoración, si se confirma la existencia de criterios suficientes para el diagnóstico, se recomienda la derivación desde pediatría a salud mental. Por tanto, aunque la cumplimentación del protocolo no se da en todos los casos, ni el acuerdo diagnóstico con los orientadores es total, en un porcentaje importante de los casos, la derivación del pediatra se basa en una sospecha diagnóstica del centro escolar.

En cuanto a la calidad de los diagnósticos realizados en pediatría, consideran- do como válido el criterio diagnóstico de salud mental, se puede afirmar que a nivel general su capacidad para detectar los trastornos es buena, aunque existen algunas excepciones. Así, en la amplia mayoría de los trastornos la especificidad del diagnóstico de los pediatras es muy elevada, ya que en 9 de las 14 categorías se confirma el diagnóstico de pediatría al menos en $2 / 3$ de los casos, mientras que en 3 de ellas, se confirma en al menos la mitad de los casos. Así, el porcentaje de falsos positivos en la mayoría de los diagnósticos es muy reducido. De hecho, en la totalidad de los casos identificados como Trastorno Generalizado del Desarrollo y Trastornos de la eliminación (enuresis y encopresis) se confirma el diagnóstico efectuado en atención primaria (100\%). La excepción se da en los trastornos específicos del desarrollo, los trastornos depresivos y especialmente en los adaptativos en los que la coincidencia con el diagnóstico de pediatría es menos frecuente (en el $54 \%$ en los dos primeros y en el $36 \%$ ). En cuanto a los trastornos psicóticos, la escasa incidencia ( 2 casos) impide valorar con fiabilidad la capacidad de detección de este tipo de cuadros.

Respecto a la capacidad para detectar el trastorno presente, a nivel general, se puede señalar que la sensibilidad del diagnóstico de los pediatras, fue inferior a la especificidad. De hecho, para la mitad de las categorías diagnósticas la detección del trastorno no superó el $50 \%$ de los casos, aunque de nuevo, existe una importante variabilidad en función del trastorno. Por un lado, se detectan como tales el 100\% de los trastornos de la conducta alimentaria, el $92 \%$ de los trastornos de ansiedad, el $87 \%$ de los trastornos de la eliminación y el $83 \%$ de los TDAH. En el otro extremo, la capacidad de detección de los trastornos específicos del desarrollo (37\%) y de los códigos Z (27\%) resulta muy inferior al resto de diagnósticos, lo que refleja un incremento de los falsos negativos. Hay que considerar además, que estos porcentajes se hallan sobre el total de los casos derivados, obviando aquellos pacientes valorados en la consulta de pediatría que no son derivados a salud mental. Por tanto, tan sólo se pue- 
den considerar como medidas aproximadas de la sensibilidad y especificidad.

Por tanto, entre los trastornos con menor concordancia, además de los ya comentados trastornos adaptativos, se encuentran los trastornos específicos del desarrollo y los trastornos depresivos. Dentro de la primera categoría, son posiblemente los trastornos específicos del desarrollo del aprendizaje escolar y especialmente, el trastorno del aprendizaje de la lectura y de la escritura, los más prevalentes. Dado que el diagnóstico de este trastorno es difícil de determinar sin aplicar pruebas psicopedagógicas específicas es lógico que su diagnóstico se pase por alto desde la consulta de pediatría. Además, a pesar de que estos trastornos aparecen como categoría diagnóstica en los manuales al uso y de que frecuentemente se acompañan de alteraciones psicopatológicas en distinto grado, presentan características diferenciales y no pueden considerarse completamente equiparables a otros trastornos mentales. Por tanto, no puede considerarse como indicador de mal funcionamiento la ausencia de detección de este tipo de patología desde atención primaria.

En lo referente a los trastornos depresivos su infradiagnóstico desde pediatría puede deberse a varios motivos. Por un lado, durante años se ha negado la existencia de este cuadro en la infancia ${ }^{18}$, por lo que los profesionales pueden estar menos predispuestos a observar esta sintomatología. Por otro lado, aunque los trastornos depresivos en la infancia y adolescencia comparten síntomas con los del adulto, son más frecuentes las formas atípicas ${ }^{9}$ y la presentación puede variar en función del estadio evolutivo, lo que probablemente dificulta su detección.

A la hora de interpretar los resultados de este estudio es importante considerar algunas limitaciones en el diseño. En primer lugar, con el objetivo de facilitar el análisis de los datos se consideraron categorías amplias de trastornos, como por ejemplo trastornos de ansiedad, en vez de categorías específicas, como fobias o ansiedad de separación. En segundo lugar, se analizaron los datos que constaban en los volantes de derivación como diagnósticos y no como síntomas, y es posible que esto no refleje la intención del profesional que completó el informe. En tercer lugar, para simplificar el análisis de la concordancia se consideró únicamente el diagnóstico principal, descartando los diagnósticos comórbidos cuando existían. Por último, hay que señalar que el estudio se ha llevado a cabo basándose en los diagnósticos realizados por tan sólo dos psicólogas clínicas (las profesionales del centro que realizaron todas las primeras consultas) y por tanto, los resultados obtenidos pueden estar excesivamente influidos por las características de estas profesionales. No obstante, los diagnósticos se realizaron siguiendo los criterios diagnósticos CIE-10, lo que aumenta su fiabilidad y disminuye las variaciones entre profesionales.

En definitiva, el nivel de concordancia en el diagnóstico alcanzado entre los servicios de pediatría y el centro de Salud Mental es bueno. Este dato es un indicador positivo respecto al nivel de coordinación alcanzado entre ambos niveles asistenciales y refleja asimismo la tendencia a la intervención desde una perspectiva multidisciplinar desde la que la coordinación con el centro escolar (y en concreto con la figura del orientador) resulta fundamental. Sin duda, la coincidencia en el diagnóstico entre ambos profesionales va a facilitar la coordinación en el trabajo de ambos, favoreciendo por ejemplo, el adecuado seguimiento por parte de pediatría de las pautas de tratamiento indicadas en salud mental. No es posible asegurar que un mejor nivel de coordinación incida en la calidad de los tratamientos o en el pronóstico del caso, pero sin duda facilita la comprensión de los problemas de salud mental y el cumplimiento del tratamiento para los pacientes y sus familias. Por otra parte, el hecho de que los pediatras sean capaces de valorar adecuadamente los trastornos mentales, va a posibilitar su identificación precoz y agilizará la derivación a salud mental y la instauración del tratamiento pertinente y esto previsiblemente mejorará el pronóstico. 


\section{BIBLIOGRAFÍA}

1. World Health Organization. Prevention of Mental Disorders. Effective Interventions And Policy Options. Summary Report. Geneva 2004. [consultado el 7-1-2009]: Disponible en: http://whqlibdoc.who.int/ publications/2004/924159215X.pdf

2. Roberts RE, Attkisson CC, Rosenblatt A. Prevalence of psychopathology among children and adolescents. Am J Psychiatry 1998; 155: 715-725.

3. Morán Sánchez I, Navarro-Mateu F, Robles Sanchez F, Concepción Salesa A. Validez del diagnóstico clínico de trastorno por déficit de atención con hiperactividad en las derivaciones de pediatría a la consulta de psiquiatría infantil. Aten Primaria 2008; 40: 29-33.

4. Kelleher KJ, Campo JV, Gardner P. Management of pediatric mental disorders in primary care: where are we know and where are we going? Curr Opin Pediatr 2006; 18: 649-653.

5. Williams J, Klinepter K, Palmes G, Pulley A, Foy JM. Diagnosis and treatment of behavioural health disorders in pediatric practice. Pediatrics 2004; 114: 601-606.

6. Goldberg ID, Roghmann KJ, Mclnerny TK, BuRKe JD. Mental Health problems among children seen in pediatric practice: prevalence and management. Pediatrics 1984; 73: 278-293.

7. Rodríguez HeRnandez PJ. Trastornos psiquiátricos infantiles de etiología neurobiológica y su despistaje en Atención Primaria. Can Ped 2006; 30: 9-20.

8. Bernal, P. Hidden morbidity in Pediatric Primary Care. Pediatr Ann 2003; 32: 413-418.

9. Organización Mundial de la Salud CIE-10. Clasificación de los trastornos mentales y del comportamiento. Madrid: Méditor, 1992.
10. American Psychiatric Association Diagnostic and statistical manual of mental disorders $4^{\text {th }}$ ed rev. Washington D.C.: APA, 2000.

11. Landa González N, GoÑ Sarriés A, García de JaLón Aramayo E, Lizasoin URRa E. Concordancia en el diagnóstico entre Atención Primaria y Salud Mental. Aten Primaria 2008; 40: 285290.

12. García Testal A, Sancho Blasco F, Julve Pardo R, Puche Pinazo E, Rabanaque Mallén G. Estudio de las derivaciones de atención primaria a salud mental: ¿qué coincidencia existe entre los motivos de derivación y el diagnóstico del especialista? Aten Primaria 1998; 22: 233-238.

13. HerRán A, López-Lanza JR, Ganzo H, Cadiñanos A, Dízz-Manrique JE, Vázquez-Barquero JL. Derivación de los pacientes con enfermedad mental desde Atención Primaria a Salud Mental. Actas Esp Psiquiatr 2000; 28: 13-21.

14. Miranda Chueca I, Peñarrubia María MT, García Bayo I, Caramés Durán E, Soler Vila M, Serrano Blanco A. ¿Cómo derivamos a salud mental desde atención primaria? Aten Primaria 2003; 32: 524-530.

15. Altman DG. Practical statistics for medical research. New York: Chapman and Hall, 1991.

16. Polanczyk G, Jensen P. Epidemiologic considerations in Attention Deficit Hyperactivity disorder: a review and update. Child Adolesc Psychiatric Clin N Am 2008; 17: 245-260.

17. Rowland AS, Lessene CA, Abramowitz AJ. The epidemiology of attencion-deficit/hyperactivity disorder (ADHD): a public health view. Ment Retard Dev Disabel Res Rev 2002; 8: 162-170.

18. JimÉNEZ HeRnÁNDEZ M. Depresión infantil: aspectos clínicos. En: Jiménez Hernández M editor. Psicopatología infantil, Málaga: Ed. Aljibe, 1997: 99-118. 